\title{
Innovation and Environmental Policy: Clean vs. Dirty Technical Change
}

\author{
Maria A. Cunha-e-Sá ${ }^{a}$, and Alexandra Leitão ${ }^{b,},{ }^{*}$,and Ana B. Reis ${ }^{a}$ \\ ${ }^{a}$ Universidade Nova de Lisboa, Faculdade de Economia, \\ Campus de Campolide, PT-1099-032, Portugal \\ ${ }^{b}$ Universidade Católica Portuguesa, Centro Regional do Porto, \\ Faculdade de Economia e Gestão, \\ Rua Diogo Botelho, 1327, PT-4169-005, Portugal
}

March 2, 2010

\begin{abstract}
We study a two sector endogenous growth model with environmental quality with two goods and two factors of production, one clean and one dirty. Technological change creates clean or dirty innovations. We compare the laissez-faire equilibrium and the social optimum and study first- and second-best policies. Optimal policy encourages research toward clean technologies. In a second-best world, we claim that a portfolio that includes a tax on the polluting good combined with optimal innovation subsidy policies is less costly than increasing the price of the polluting good alone. Moreover, a discriminating innovation subsidy policy is preferable to a non-discriminating one.

Keywords: pollution; endogenous growth; innovation; environmental policy; laissez-faire equilibrium; optimal equilibrium; discriminating vs. non-discriminating subsidies to R\&D;
\end{abstract}

JEL codes: H23; O3; O41

\footnotetext{
${ }^{*}$ Corresponding author: Rua Comendador Sá Couto, no 55 - $2^{\circ}$ Dto., 4520-192 Santa Maria da Feira, Portugal; Phone: +351 91 4891810; Fax: +351 256 381308; E-mail: apleitao@netcabo.pt.
} 


\section{Introduction}

The reduction of greenhouse gas emissions makes technological change and the development of renewable energy central issues, with important implications for policy design. Some policies, such as a tax on emissions or a tradable emissions permit system, make emissions expensive. Other policies focus on reducing the cost of $R \& D$. However, not all technological change targets pollution savings. For instance, technological change in fossil-fuel (e.g., oil) extraction industries has definitely contributed to both the discovery of new sources, as is recently the case in Brazil, and to the possibility of using non-conventional oil sources such as tar sands, with no particular emphasis on environmental friendliness. At the same time, a great deal of focus has been placed on the role of innovation in lowering the cost of carbon-free sources. $^{1}$

In this context, the contribution of this paper is to study environmental and technology policies in a two sector endogenous growth model where the two types of technological change - clean and dirty - are present, which allows us to consider not only the usual R\&D distortions discussed by Stokey [18] but also the role of $R \& D$ in internalizing the pollution externality and its policy implications. There is evidence that in a market economy research tends to be under-provided relative to the social optimum (Jones and Williams [13], [14]). However, this result does not take into account the effect of growth on environmental quality. Our concern goes to how environmental and technology policies affect both the rate and the type of technological

\footnotetext{
${ }^{1}$ Carraro and Siniscalco [5] present studies showing that large corporations typically adjust to environmental policy measures through innovation, rather than by switching inputs or reducing output. They also mention the fact that without innovation very high taxes are required to curb $\mathrm{CO}_{2}$ emissions.
} 
change. The question is not only, "are there limits to growth?" as in Stokey [19], but also in the kind of innovations we are interested in and the incentives policy should give. The extent to which research is environmentally oriented is a matter of discussion, as are its policy implications.

We follow the work of Acemoglu [1] and Acemoglu and Zilibotti [2], where two types of innovation are carried out by profit maximizing firms, and extend it to include the environmental dimension. We consider two goods and two factors of production, one clean and one dirty. Pollution is a by-product of the dirty-intensive good production, which affects environmental quality and negatively influences consumers' utility. There are two different $R \& D$ sectors associated with each sector of production. $R \& D$ firms develop new clean or dirty complementary intermediates, depending on relative profitability.

Our purpose is to study the impact of policy-induced changes on the rate of innovation and the type of technical change. To this end, we focus on the induced impact on the relative productivity of dirty and clean technologies, a measure of the dirty-bias in the economy. ${ }^{2}$ We study the implementation of the social optimum. First- and second-best policies are examined, mainly focusing on the consequences of the composition of innovation.

In the laissez-faire economy, the research effort directed at new dirty complementary intermediates when compared to the one devoted to new clean intermediates is relatively too high. Therefore, technology is too dirtybiased and emissions intensity of final output is too high.

There are three distortions in our economy: the environmental externality, the surplus appropriability problem of the research sector, and the

\footnotetext{
${ }^{2}$ This is in contrast to Acemoglu's [1] biased technical change, where the relative factor rewards respond to changes in the relative endowments.
} 
monopoly power of intermediate inputs' producers. In a first-best world, three policies are needed to optimally address the three market failures: environmental policy, subsidies for research, and subsidies for the monopolists. We compute the optimal values of these economic policy tools. We show that the optimal policy implements the optimal rate of innovation and simultaneously encourages a change in the quality of research towards the clean sector of the economy.

Finally, we study second-best policy, which depends on the policy-maker's ultimate goal. As expected, we show that implementing the efficient dirtybias only through emissions pricing policies has such a strong negative impact on economic activity that such policies have little political appeal. This result suggests that in the presence of a pollution externality there is an additional rationale to promote $\mathrm{R} \& \mathrm{D}$. In this context, if the regulator chooses to subsidize innovation, we show that while a non-discriminating policy between the dirty and clean sectors has no impact on the level of emissions intensity, keeping it at the laissez-faire level, independently of the subsidy rate, when the subsidies are different emissions intensity decreases. However, in order to implement the efficient level of emissions, an additional tax on the polluting good is needed.

In endogenous growth models with environmental concerns, stagnation along the optimal path is avoided, either through increased abatement or through technological progress, as shown in Bovenberg and Smulders [4], Stokey [19], Elbasha and Roe [7], and Reis [16], among others. Therefore, the development of environmentally- friendly technologies protects the environment and, at the same time, drives economic growth, lowering the trade-off between environmental quality and growth. Grimaud [9] has also 
focused on the importance of endogenous research in market economies with environmental damages. Our paper extends this literature since we consider two different R\&D types.

Ricci [17] and Hart [11], [12], analyze, in a vintage model, the effects of environmental regulation on the quantity of research effort, and on its quality (allocation of research inputs between research sectors). However, we follow a different framework that focuses primarily on the relative productivity of dirty and clean technologies.

More recently, Grimaud and Rouge [10] study an endogenous growth model with a polluting and a non-polluting resource with specific research sectors. These authors study the effects of optimal economic policy on the reallocation of research efforts across the two sectors. In contrast to our paper, Grimaud and Rouge focus only on first-best policy. In a secondbest context, our results also support the arguments of Montgomery and Smith [15] and Fisher and Newell [8], although the context is different from ours in both cases. As claimed by these authors, an emissions price high enough to induce the needed innovation cannot be credibly implemented and, therefore, $R \& D$ is the key for dealing with climate change. In this context, a portfolio that includes a tax on the polluting good combined with optimal innovation subsidy policies is less expensive than one that increases the price of the polluting good alone. We further extend this result by showing that a discriminating innovation subsidy policy is preferable to a non-discriminating one.

The paper is organized as follows. The model is presented in Section 2. The regulated equilibrium is derived in Section 3. In Section 4, the solution to the social planner's problem is discussed and compared to the laissez-faire 
solution. Section 5 focuses on policy implications. Section 6 summarizes the main conclusions. Technical details are presented in the appendices.

\section{The Model}

The economy is closed and produces a final output from two commodities, which use primary factors of production and a set of differentiated intermediate inputs. The model builds upon Acemoglu [1] and Acemoglu and Zilibotti [2], introducing preferences for environmental quality.

Consumers There are many identical infinitely lived consumers who get utility from consumption of final output and environmental quality. Utility of a representative consumer is given by

$$
U=\int_{0}^{\infty}(\log C+\mu \log Q) e^{-\rho t} d t
$$

where $C$ is consumption of the final output, $Q$ measures the quality of the environment, $\mu$ reflects environmental preferences and $\rho$ is the rate of time preference. The utility function is increasing and strictly concave in $C$ and $Q$ as long as $\mu>0$. The representative consumer values consumption more than environmental quality, such that $\mu<1$. Consumers are endowed with two primary factors of production: a clean $(L)$ one and a dirty $(Z)$ one. ${ }^{3}$

Final output sector The final output $Y$ is produced from two goods, $Y_{L}$ and $Y_{Z}$, through a Cobb-Douglas production function, as in Acemoglu and Zilibotti $[2]^{4}$

$$
Y=Y_{L}^{\gamma} Y_{Z}^{1-\gamma}
$$

\footnotetext{
${ }^{3}$ As in Acemoglu [1] and Acemoglu and Zilibotti [2], in order to simplify the analysis we do not allow factor accumulation.

${ }^{4}$ Acemoglu [1] considers two types of goods but the elasticity of substitution between them is different from one. Acemoglu and Zilibotti [2] consider a Cobb-Douglas with a continuous of goods. The fact that we assume an elasticity of substitution of 1 implies that we focus only on the relative physical productivity of dirty and clean factors and not on the corresponding relative factor rewards. Thus, according to Acemoglu's [1] terminology, we focus on factor-augmenting technical change and not on factor-biased technical change.
} 
$Y_{L}$ is intensive in the clean factor, $L$, while $Y_{Z}$ uses the dirty factor, $Z$, intensively. ${ }^{5} Y_{L}$ and $Y_{Z}$ production functions are given by

$Y_{L}=\frac{1}{1-\beta}\left(\int_{0}^{N_{L}} x_{L}(j)^{1-\beta} d j\right) L^{\beta} \quad$ and $\quad Y_{Z}=\frac{1}{1-\beta}\left(\int_{0}^{N_{Z}} x_{Z}(j)^{1-\beta} d j\right) Z^{\beta}$

where $\beta \in(0,1)$, and $L$ and $Z$ are assumed to be supplied inelastically.

The $L$-intensive good is produced from the clean factor and a range of $L$ complementary ( $L$-augmenting) intermediates $\left(x_{L}\right)$. The range of intermediate inputs of type $x_{L}$ available at a given time period, is denoted by $N_{L}$. The amount of input $j$ used is denoted by $x_{L}(j)$. The production function for $Y_{Z}$ uses the dirty factor intensively and $Z$-complementary intermediates $\left(x_{Z}\right) . N_{Z}$ and $x_{Z}(j)$ are similar.

The ratio $\left(N_{Z} / N_{L}\right)$ determines the relative productivity of dirty and clean technologies, and measures the dirty-bias in the economy.

The markets for $Y_{L}$ and $Y_{Z}$ are assumed to be perfectly competitive.

Intermediate inputs sectors Each input $j \in\left[0, N_{L}\right]$ and $j \in\left[0, N_{Z}\right]$ is supplied by a monopolist who faces a marginal cost, $\psi$, in terms of the final good, of producing intermediate inputs, which is the same for all intermediate inputs. The monopolist sets the price for the intermediate input, $\chi_{L}(j)$ or $\chi_{Z}(j)$.

RED sector Technical change is modeled as the invention of new dirty and clean intermediates. It is assumed that only the final good is used as an input in this sector. The production functions for the innovations are

$$
\dot{N}_{L}=\eta_{L} R_{L} \quad \text { and } \quad \dot{N}_{Z}=\eta_{Z} R_{Z}
$$

\footnotetext{
${ }^{5}$ For example, we can think of $Y$ as the production of energy, with $Y_{L}$ as the nonemitting renewable energy sector and $Y_{Z}$ as emitting fossil-fueled energy. According to this example, the model incorporates innovation in both sectors. While innovation increases renewable energy efficiency by augmenting the productivity of renewables, it is reasonable to assume that fossil-fueled technologies will also innovate, but are not necessarily pollution-saving.
} 
where $R_{L}$ and $R_{Z}$ are spending on $\mathrm{R} \& \mathrm{D}$ for the clean and dirty factorintensive good, respectively. One unit of final good spent on R\&D directed at $L$-complementary intermediates will generate $\eta_{L}$ new varieties of clean intermediates. $\dot{N}_{Z}$ is explained similarly.

Research is motivated by the future benefits that emerge from the discovery of a new variety. A firm that discovers a new variety receives a patent on this intermediate input and becomes a monopolist. Thus, R\&D firms develop new clean or dirty intermediates depending on relative profitability.

Budget constraint The budget constraint of the economy is

$$
C+I+R=Y
$$

that is,

$$
C+\left[\psi \int_{0}^{N_{L}} x_{L}(j) d j+\psi \int_{0}^{N_{Z}} x_{Z}(j) d j\right]+\left(R_{L}+R_{Z}\right)=Y
$$

where $I$ denotes investment, and $R$ is total $\mathrm{R} \& \mathrm{D}$ expenditure.

Environmental quality We model the quality of the environment as a flow variable. Environmental damages are a by-product of the dirty sector. Environmental quality is measured by the inverse of emissions, $E_{Z}$,

$$
Q=\frac{1}{E_{Z}}=\frac{1}{\delta Y_{Z}}, \quad 0<\delta<1
$$

which are proportional to the production of $Y_{Z}$, and $\delta$ is a technology parameter that quantifies the detrimental effect of $Y_{Z}$ on the environment. When studying environmental policy we focus on emissions intensity of final output, $\frac{E_{Z}}{Y} \cdot 6$

\footnotetext{
${ }^{6}$ This approach is followed, for example, by Canada, which has implemented a domestic trading system in which emission reduction credits can be traded to limit carbon emissions from much of the economy's energy and industrial sectors. The emission targets are expressed in terms of a reduction in emission intensity rather than in absolute levels of emissions reductions (Amano and Sedjo [3]).
} 


\section{Equilibrium in the Regulated Economy}

In this section we derive the equilibrium with government intervention. There are three distortions in this economy: (i) the surplus appropriability problem tends to generate too little research, (ii) intermediate goods are produced by monopolists, producing less than optimally, and finally, (iii) because of the environmental externality, production of $Y_{Z}$ is larger than optimal. We assume that the government is able to address all imperfections.

We consider the following set of instruments: (i) a proportional subsidy, $\phi>1$, paid by the government to the R\&D sector for each new design, which is assumed to be the same for new $L$ and $Z$-complementary intermediates. This subsidy decreases the costs of a new variety to $1 /\left(\phi \eta_{i}\right), i=L, Z$, (ii) a subsidy, $s$, paid by the government to each producer of intermediate inputs, and finally, (iii) a tax, $\tau$, levied on the polluting sector, proportional to the emissions of the dirty good.

Firms in the clean sector maximize profits, choosing $L$ and $x_{L}(j)$, taking as given the price of their product, $p_{L}$, the price of the primary factor, $\omega_{L}$, the prices of the intermediate inputs, $\chi_{L}(j)$, and the range of intermediate inputs, $N_{L}$. Firms in the dirty sector pay the environmental tax and face a similar profit maximization problem.

From the first-order conditions of these problems, the rewards for the primary factors of production, $\omega_{L}$ and $\omega_{Z}$, are obtained, as follows

$$
\omega_{L}=\beta \frac{p_{L} Y_{L}}{L} \quad \text { and } \quad \omega_{Z}=\beta \frac{p_{Z}(1-\tau \delta) Y_{Z}}{Z},
$$

and the demand for each intermediate input is given by

$$
x_{L}(j)=\left(\frac{p_{L}}{\chi_{L}(j)}\right)^{\frac{1}{\beta}} L \quad \text { and } \quad x_{Z}(j)=\left(\frac{p_{Z}(1-\tau \delta)}{\chi_{Z}(j)}\right)^{\frac{1}{\beta}} Z
$$


The demand for each intermediate input is the same for all $j \in\left[0, N_{L}\right]$ and for all $j \in\left[0, N_{Z}\right]$.

Each monopolist in the intermediate goods sectors chooses prices, $\chi_{L}(j)$ and $\chi_{Z}(j)$, that maximize profits, taking into account the demand functions in (8) and the subsidy paid by the government. Profits are maximized by $\chi_{L}(j)=\chi_{Z}(j)=\frac{\psi(1-s)}{1-\beta}, j \in\left[0, N_{L}\right]$ and $j \in\left[0, N_{Z}\right]$. To simplify the algebra, we normalize the marginal cost to $\psi=1-\beta$. In equilibrium, prices are $\chi_{L}(j)=\chi_{Z}(j)=1-s$.

Therefore, price, quantity, and, hence, the level of profits are the same for all firms operating in each intermediate inputs sector. Using the prices and demands above, the monopolists' profits are

$$
\pi_{L}=\beta(1-s)\left(\frac{p_{L}}{1-s}\right)^{\frac{1}{\beta}} L \quad \text { and } \quad \pi_{Z}=\beta(1-s)\left(\frac{p_{Z}(1-\tau \delta)}{1-s}\right)^{\frac{1}{\beta}} Z
$$

R\&D firms develop new clean or dirty intermediates depending on the relative profitability given by the net present value of profits.

To maintain asset market equilibrium, the rate of return from holding equities must be equal to the rate of return on a one period loan, that is,

$$
\frac{\pi_{L}}{V_{L}}+\frac{\dot{V}_{L}}{V_{L}}=r \quad \text { and } \quad \frac{\pi_{Z}}{V_{Z}}+\frac{\dot{V}_{Z}}{V_{Z}}=r
$$

These equations relate the present value of future profits, $V$ (the value of a firm in the intermediate inputs sector), to the flow of profits, $\pi$.

We will focus on the long-run balanced growth path of this economy, where prices and the interest rate are constant. Thus, the $\dot{V}$ terms are zero. Then, combining (9) and (10), yields

$$
V_{L}=\frac{\beta(1-s)\left(\frac{p_{L}}{1-s}\right)^{\frac{1}{\beta}} L}{r} \quad \text { and } \quad V_{Z}=\frac{\beta(1-s)\left(\frac{p_{Z}(1-\tau \delta)}{1-s}\right)^{\frac{1}{\beta}} Z}{r}
$$


The greater $V_{L}$ is relative to $V_{Z}$, the greater are the incentives to develop clean intermediates rather than dirty ones.

There is free entry and exit in the R\&D sector. Thus, in equilibrium, the value of a firm in the intermediate inputs sector $\left(V_{i}, i=L, Z\right)$ must be equal to the cost of a new variety $\left(\frac{1}{\phi \eta_{i}}, i=L, Z\right)$.

We assume a balanced growth path (BGP), where prices $p_{L}$ and $p_{Z}$ are constant, and $N_{L}$ and $N_{Z}$ grow at the same constant rate. In equilibrium, along a BGP, all variables grow at the same rate: $\dot{Y} / Y=\dot{N}_{L} / N_{L}=$ $\dot{N}_{Z} / N_{Z}=\dot{C} / C=\dot{Y}_{Z} / Y_{Z}=\dot{Y}_{L} / Y_{L}=g$. In order to generate growth, the number of new designs must be expanding over time. This occurs if the spending on R\&D is increasing. More spending means more ideas, sustaining growth. In this case, the growth in ideas is clearly related to the final output growth $\left(\dot{R}_{L} / R_{L}=\dot{R}_{Z} / R_{Z}=g\right)$.

There will be innovation in both sectors if the following condition holds

$$
\phi \eta_{L} V_{L}=\phi \eta_{Z} V_{Z} \quad \text { or } \quad \phi \eta_{L} \pi_{L}=\phi \eta_{Z} \pi_{Z}
$$

According to this condition, it is equally profitable to invest in the development of $L$ and $Z$-complementary intermediate inputs, so that along the BGP, $N_{L}$ and $N_{Z}$ can both grow. After substitution of (11), it implies

$$
\frac{\eta_{Z}}{\eta_{L}}\left(\frac{p_{Z}(1-\tau \delta)}{p_{L}}\right)^{\frac{1}{\beta}} \frac{Z}{L}=1
$$

This condition can be solved for the dirty-bias in the regulated economy, $\left(N_{Z} / N_{L}\right)$

Substituting (8) into the production functions (3) we obtain the market productions of $Y_{L}$ and $Y_{Z}$, given $N_{L}$ and $N_{Z}$. The markets for $Y_{L}$ and $Y_{Z}$ are competitive, so market clearing implies that their relative price $p$ is given 
by

$$
p=\frac{p_{Z}}{p_{L}}=\frac{\frac{\partial Y}{\partial Y_{Z}}}{\frac{\partial Y}{\partial Y_{L}}} \text { where } \frac{\frac{\partial Y}{\partial Y_{Z}}}{\frac{\partial Y}{\partial Y_{L}}}=\frac{1-\gamma}{\gamma}\left(\frac{Y_{Z}}{Y_{L}}\right)^{-1}
$$

Substituting for $Y_{L}$ and $Y_{Z}$ and solving for $p$ we get the relative price of the two goods as a function of the environmental tax, of the relative productivity of dirty and clean technologies $\left(N_{Z} / N_{L}\right)$, and of the relative factor supply, $Z / L$ :

$$
p=(1-\tau \delta)^{-(1-\beta)}\left(\frac{1-\gamma}{\gamma}\right)^{\beta}\left(\frac{N_{Z} Z}{N_{L} L}\right)^{-\beta}
$$

From (8) and (15), the relative demand of intermediate inputs as a function of $\left(N_{Z} / N_{L}\right)$ is

$$
\left(\frac{x_{Z}}{x_{L}}\right)^{R E}=(1-\tau \delta)\left(\frac{1-\gamma}{\gamma}\right)\left(\frac{N_{Z}}{N_{L}}\right)^{-1}
$$

Substituting (15) in (13), and solving for $\left(N_{Z} / N_{L}\right)$ we obtain

$$
\left(\frac{N_{Z}}{N_{L}}\right)^{R E}=(1-\tau \delta)\left(\frac{\eta_{Z}}{\eta_{L}}\right)\left(\frac{1-\gamma}{\gamma}\right)
$$

where RE stands for the regulated economy. This equation defines the relative physical productivity of dirty versus clean factors along the BGP, which is positively affected by the relative productivity of the R\&D labs, $\frac{\eta_{Z}}{\eta_{L}}$, and the relative weight in the production of $Y, \frac{1-\gamma}{\gamma}$, and is negatively affected by the environmental tax, $\tau$.

Substituting (17) in (16), we obtain the relative demand of intermediate inputs with endogenous technology

$$
\left(\frac{x_{Z}}{x_{L}}\right)^{R E}=\left(\frac{\eta_{Z}}{\eta_{L}}\right)^{-1}
$$

From the maximization of (1), we obtain $\frac{\dot{C}}{C}=r-\rho$. Along a BGP, all variables grow at the same rate, $g=r-\rho$. This long-run growth rate 
is obtained in the following Proposition, in terms of the parameters of the model, the endowments of the economy, and the policy instruments. ${ }^{7}$

Proposition 1 The growth rate of the regulated economy at the BGP is given by $g^{R E}=\phi \beta(1-s)\left(\frac{1}{1-s}\right)^{\frac{1}{\beta}}\left(\gamma^{\gamma}(1-\gamma)^{1-\gamma}\right)^{\frac{1}{\beta}}(1-\tau \delta)^{\frac{1-\gamma}{\beta}}\left(\eta_{Z} Z\right)^{1-\gamma}\left(\eta_{L} L\right)^{\gamma}-\rho$

Proof. The free-entry condition in $R \mathscr{E} D, V_{L}=\frac{1}{\phi \eta_{L}}$, together with (11), implies $\phi \eta_{L} \beta(1-s)\left(\frac{p_{L}}{1-s}\right)^{\frac{1}{\beta}} L=r$, in the steady state. Also, assuming the price of the final good as the numéraire, the following relationship between the prices of the two goods holds $\frac{p_{L}^{\gamma} p_{Z}^{1-\gamma}}{\gamma^{\gamma}(1-\gamma)^{1-\gamma}}=1$. Solving this condition for $p_{L}$ and substituting it in the free entry condition together with (15), we obtain the growth rate of the economy for a given $\left(N_{Z} / N_{L}\right)$,

$g=\phi \eta_{L} \beta(1-s)\left(\frac{1}{1-s}\right)^{\frac{1}{\beta}}\left(\gamma^{\gamma}(1-\gamma)^{1-\gamma}\right)^{\frac{1}{\beta}}(1-\tau \delta)^{\frac{(1-\gamma)(1-\beta)}{\beta}}\left(\frac{\gamma}{1-\gamma}\right)^{1-\gamma}\left(\frac{N_{Z} Z}{N_{L} L}\right)^{1-\gamma} L-\rho$

Combining (17) with (20), the result follows.

In the next section we solve for the social planner's problem and compare the optimal solution with the laissez-faire economy, which may be obtained from (16), (17) and (19) when $\phi=1$ and $s=\tau=0$.

\footnotetext{
${ }^{7}$ We briefly analyze the stability properties of the model outside the BGP: the equilibrium condition for the relative profitability of innovations along the BGP is given by $\frac{\eta_{Z} V_{Z}}{\eta_{L} V_{L}}=1$ (see $\left.(12)\right)$. If $\frac{\eta_{Z} V_{Z}}{\eta_{L} V_{L}}>1(<1) \mathrm{R} \& \mathrm{D}$ will be undertaken only in dirty complementary (clean complementary) intermediates. Since the relative profitability of creating dirty complementary intermediates $\frac{V_{Z}}{V_{L}}$ is decreasing in $\frac{N_{Z}}{N_{L}}$ (from (11) and (15)), the system is stable.
} 


\section{Social Optimum}

The social planner's problem for this economy can be stated as

$$
\begin{gathered}
\quad \operatorname{Max} \int_{0}^{\infty}(\log C+\mu \log Q) e^{-\rho t} d t \\
\text { s.t. }(2),(3),(4),(5),(6) \\
L=\bar{L} ; \quad Z=\bar{Z} \text { and } N_{L}(0), N_{Z}(0)>0 \text { given }
\end{gathered}
$$

Thus, the current value Hamiltonian for the social planner's problem is

$$
\begin{aligned}
H= & {[\log C+\mu \log Q]+p_{C}\left[Y_{L}^{\gamma} Y_{Z}^{1-\gamma}-I-R_{L}-R_{Z}-C\right] } \\
& +r_{L}\left[\frac{1}{1-\beta}\left(\int_{0}^{N_{L}} x_{L}(j)^{1-\beta} d j\right) L^{\beta}-Y_{L}\right] \\
& +r_{Z}\left[\frac{1}{1-\beta}\left(\int_{0}^{N_{Z}} x_{Z}(j)^{1-\beta} d j\right) Z^{\beta}-Y_{Z}\right] \\
& +p_{I}\left[\psi \int_{0}^{N_{L}} x_{L}(j) d j+\psi \int_{0}^{N_{Z}} x_{Z}(j) d j-I\right] \\
& +\lambda_{L} \eta_{L} R_{L}+\lambda_{Z} \eta_{Z} R_{Z}+p_{Q}\left[\left(\delta Y_{Z}\right)^{-1}-Q\right]
\end{aligned}
$$

where $p_{C}, r_{L}, r_{Z}, p_{I}, \lambda_{L}, \lambda_{Z}, p_{Q}$ denote the shadow prices corresponding to the relevant constraints.

Solving for the social planner's problem we obtain the efficient dirtybias which is characterized in the next Proposition, where $L F$ stands for laissez-faire.

Proposition 2 The efficient dirty-bias in the economy is

$$
\begin{aligned}
& \left(\frac{N_{Z}}{N_{L}}\right)^{*}=\kappa\left(\frac{\eta_{Z}}{\eta_{L}}\right)\left(\frac{1-\gamma}{\gamma}\right)=\kappa\left(\frac{N_{Z}}{N_{L}}\right)^{L F} \\
& \text { where } \quad \kappa=\left(1-\frac{\mu C}{\frac{\partial Y}{\partial Y_{Z}} Y_{Z}}\right), \quad 0<\kappa \leqslant 1
\end{aligned}
$$

represents the pollution externality. Since $\kappa<1$, the efficient dirty-bias is smaller than the equilibrium one of the laissez-faire economy, given by (17) for $\tau=0$. 
Proof. The first-order conditions for a maximum are given by

$$
\begin{aligned}
\frac{\partial H}{\partial Y_{L}} & =p_{C} \frac{\partial Y}{\partial Y_{L}}-r_{L}=0 \\
\frac{\partial H}{\partial Y_{Z}} & =p_{C} \frac{\partial Y}{\partial Y_{Z}}-p_{Q} \frac{Q}{Y_{Z}}-r_{Z}=0 \\
\frac{\partial H}{\partial C} & =C^{-1}-p_{C}=0 \\
\frac{\partial H}{\partial Q} & =\mu Q^{-1}-p_{Q}=0 \\
\frac{\partial H}{\partial x_{L}(j)} & =r_{L} x_{L}(j)^{-\beta} L^{\beta}+p_{I} \psi=0 \\
\frac{\partial H}{\partial x_{Z}(j)} & =r_{Z} x_{Z}(j)^{-\beta} Z^{\beta}+p_{I} \psi=0 \\
\frac{\partial H}{\partial I} & =-p_{C}-p_{I}=0 \\
\frac{\partial H}{\partial R_{L}} & =-p_{C}+\lambda_{L} \eta_{L}=0 \\
\frac{\partial H}{\partial R_{Z}} & =-p_{C}+\lambda_{Z} \eta_{Z}=0 \\
\dot{\lambda}_{L} & =\rho \lambda_{L}-r_{L} \frac{1}{1-\beta} x_{L}^{1-\beta} L^{\beta}-p_{I} \psi x_{L} \\
\dot{\lambda}_{Z} & =\rho \lambda_{Z}-r_{Z} \frac{1}{1-\beta} x_{Z}^{1-\beta} Z^{\beta}-p_{I} \psi x_{Z}
\end{aligned}
$$

Also, the following transversality conditions must hold:

$$
\lim _{t \longrightarrow \infty} \lambda_{L} N_{L} e^{-\rho t}=0 ; \quad \lim _{t \longrightarrow \infty} \lambda_{Z} N_{Z} e^{-\rho t}=0
$$

Solving (29) and (30) for the demands of the intermediate inputs, and combining them with (31), (25), (26), (27), and (28), we obtain

$$
\begin{aligned}
& x_{L}^{*}(j)=\left(\frac{r_{L}}{p_{C} \psi}\right)^{\frac{1}{\beta}} L=\left(\frac{\frac{\partial Y}{\partial Y_{L}}}{\psi}\right)^{\frac{1}{\beta}} L \\
& x_{Z}^{*}(j)=\left(\frac{r_{Z}}{p_{C} \psi}\right)^{\frac{1}{\beta}} Z=\left(\frac{\frac{\partial Y}{\partial Y_{Z}}-\mu \frac{C}{Y_{Z}}}{\psi}\right)^{\frac{1}{\beta}} Z
\end{aligned}
$$

The optimal demand for each input is the same for all $j \in\left[0, N_{L}\right]$ and $j \in\left[0, N_{Z}\right]$, and it is constant along the BGP. ${ }^{8}$ From (26), (27), and (28),

\footnotetext{
${ }^{8}$ In the first-order conditions (34) and (35) this is already assumed.
} 
the shadow price of the $Z$-intensive good is given by $r_{Z}=\kappa p_{C} \frac{\partial Y}{\partial Y_{Z}}$. From conditions (32) and (33), $\frac{\dot{\lambda}_{L}}{\lambda_{L}}=\frac{\dot{\lambda}_{Z}}{\lambda_{Z}}$ follows. Thus, equating (34) and (35), combined with (29), (30), (32), (33), (37), and (38) implies that

$$
\frac{\eta_{Z}}{\eta_{L}}\left(\frac{r_{Z}}{r_{L}}\right)^{\frac{1}{\beta}} \frac{Z}{L}=1
$$

Substituting (37) and (38) into the production functions (3), we obtain the optimal $Y_{L}$ and $Y_{Z}$, given $N_{L}$ and $N_{Z}$. Substituting these into the relative marginal product of $Y_{Z}$, given by (14), and solving, we get the efficient marginal rate of transformation between the two goods, as a function of both the relative productivity of dirty and clean technologies, $\left(N_{Z} / N_{L}\right)$, and the relative factor supply, $(Z / L)$, as follows

$$
\frac{\frac{\partial Y}{\partial Y_{Z}}}{\frac{\partial Y}{\partial Y_{L}}}=\kappa^{-(1-\beta)}\left(\frac{1-\gamma}{\gamma}\right)^{\beta}\left(\frac{N_{Z} Z}{N_{L} L}\right)^{-\beta}
$$

Substituting (40) and $r_{L}$ and $r_{Z}$ in (39), and solving for $\left(N_{Z} / N_{L}\right)$, we get equation (23). Notice that when there is no externality, as in Acemoglu [1], $\kappa$ is equal to 1.9

This Proposition shows that the technology is too dirty-biased (higher $\left.N_{Z} / N_{L}\right)$ in the laissez-faire economy relative to the optimum. As environmental damages are external, there is too much research effort directed at new dirty complementary intermediates. Moreover, the higher the environmental concern (the smaller $\kappa$ ), the lower the efficient dirty-bias. In the next section we look at policies that reduce the dirty-bias in the economy, promoting the innovation of new clean intermediates.

From (23) it follows that in the laissez-faire economy the relative production of $Y_{Z}$ is greater than the efficient one. Consequently, the emissions

\footnotetext{
${ }^{9} \kappa$ is studied in detail in Appendix A.
} 
intensity of final output is higher than optimal. The laissez-faire pollution level is higher than optimal, as expected.

From (37), (38), and (40), it follows that the relative demand of intermediate inputs as a function of $\left(N_{Z} / N_{L}\right)$ is

$$
\left(\frac{x_{Z}}{x_{L}}\right)^{*}=\kappa\left(\frac{1-\gamma}{\gamma}\right)\left(\frac{N_{Z}}{N_{L}}\right)^{-1}
$$

Notice that for a given technology, internalizing pollution implies a decrease in the relative demand of intermediate inputs. After taking into account the endogenous technology, this demand is the same as in the laissez-faire economy (see (18)).

The long-run growth rate and the optimal innovation rate for this economy are presented in Proposition 3.

Proposition 3 The growth rate of the economy at the BGP is given by

$$
g^{*}=\left(\frac{1}{\psi}\right)^{\frac{1}{\beta}} \beta\left(\gamma^{\gamma}(1-\gamma)^{1-\gamma}\right)^{\frac{1}{\beta}} \kappa^{\frac{1-\gamma}{\beta}}\left(\eta_{Z} Z\right)^{1-\gamma}\left(\eta_{L} L\right)^{\gamma}-\rho
$$

The optimal growth rate is higher (i) the larger the country's endowment of $L$ and $Z$, (ii) the smaller the cost of new intermediate inputs (larger $\eta_{i}$, $i=L, Z$ ), (iii) the smaller the rate of time preference (smaller $\rho$ ), and, (iv) the less consumers value the environment (lower $\mu$ ).

Proof. From conditions (25), (27), (29), (32), (34), (37), together with (40), and the production function, (2), and the relative marginal product of $Y_{Z}$, given by (14), we obtain, after some algebra, the growth rate of the economy, for a given $\left(N_{Z} / N_{L}\right)$,

$g=\eta_{L}\left(\frac{1}{\psi}\right)^{\frac{1}{\beta}} \beta\left(\gamma^{\gamma}(1-\gamma)^{1-\gamma}\right)^{\frac{1}{\beta}} \kappa^{\frac{(1-\gamma)(1-\beta)}{\beta}}\left(\frac{\gamma}{1-\gamma}\right)^{1-\gamma}\left(\frac{N_{Z} Z}{N_{L} L}\right)^{1-\gamma} L-\rho$

Combining (23) with (43), the result for $g^{*}$ follows. 
Differentiating the optimal growth rate (42) with respect to the relevant parameter, the comparative static results follow.

Notice that $\kappa$ (equation (24)) is endogenously determined ( $\kappa$ is studied in detail in Appendix A). However, taking this into account does not change our results. ${ }^{10}$

In the model the impact of the environmental externality is captured by $\mu$. When consumers do not value environmental quality $(\mu=0)$, from (48), it follows that $\kappa$ is equal to 1 . When environmental quality is as important to consumers' welfare as consumption $(\mu=1)$, then $\kappa$ is negative. The more consumers value the environment (higher $\mu$, for $\mu<1$ ), and, therefore, the larger the environmental externality, the lower is $\kappa,{ }^{11}$ and the lower is the optimal growth rate of the economy. ${ }^{12}$

The environmental externality influences the growth rate through (i) the adjustment on the intermediate inputs, and (ii) the incentives to innovation. Before incorporating the efficient dirty-bias, the growth rate of the economy, given by (43), captures the externality through the adjustment on the intermediate inputs, for a given $\left(N_{Z} / N_{L}\right)$. However, the externality is only fully accounted for after incorporating the optimal dirty-bias of technology, as shown in (42).

\footnotetext{
${ }^{10}$ The comparative static results in Proposition 3 already take into account the endogeneity of $\kappa$.

${ }^{11}$ Proof in Appendix A.

${ }^{12}$ We assume a positive optimal growth rate of the economy, $g^{*}>0$, that is, $\kappa>$ $\left[\frac{\rho}{\beta A}\right]^{\frac{\beta}{1-\gamma}}$, where $A=\left(\frac{1}{\psi}\right)^{\frac{1}{\beta}}\left(\gamma^{\gamma}(1-\gamma)^{1-\gamma}\right)^{\frac{1}{\beta}}\left(\eta_{Z} Z\right)^{1-\gamma}\left(\eta_{L} L\right)^{\gamma}$. This means that $\mu<$ $\bar{\mu}<1$, where $\bar{\mu}=\frac{\left(\frac{\rho}{\beta A}\right)(1-\gamma) A\left[1-\left(\frac{\rho}{\beta A}\right)^{\frac{\beta}{1-\gamma}}\right]}{\left(\frac{\rho}{\beta A}\right)(1-\gamma) A\left[1-\left(\frac{\rho}{\beta A}\right)^{\frac{\beta}{1-\gamma}}\right]+\left(\frac{\rho}{\beta A}\right)^{\frac{\beta}{1-\gamma}}(1-\gamma) \rho+\gamma \rho}$. Therefore, the solution we are considering is only possible for environmental preferences such that $0<\mu<\bar{\mu}$. A very high environmental concern by consumers would imply either zero or negative growth. Also, from the maximization of the utility function, it has to be the case that $g^{*}<\frac{\rho}{1-\mu}$. If this condition holds for $\mu=0$, it also holds for positive values of $\mu$.
} 
The optimal growth rate, (42), internalizes both the environmental externality (through $\kappa$ ) and the monopoly and R\&D distortions (through $\left(\frac{1}{\psi}\right)^{\frac{1}{\beta}}$ ). The environmental externality $(\kappa<1)$ contributes to a decrease in $g^{*}$ relative to $g^{L F}$, while the monopoly and $\mathrm{R} \& \mathrm{D}$ distortions $\left(\left(\frac{1}{\psi}\right)^{\frac{1}{\beta}}>1\right)$ work in the opposite way. ${ }^{13}$

The laissez-faire equilibrium growth rate, $g^{L F}$, can be smaller or larger than the optimal growth rate, $g^{*}$. If the distortion for the monopoly and R\&D distortions prevails, then $g^{*}>g^{L F}$. However, the optimal growth rate is lower than it would be without environmental externalities. In such a case, when the environmental externality is not too large (large $\kappa$ ), the optimal solution is characterized by boosting growth and decreasing the dirty-bias in the economy. If the distortion for the externality prevails, then $g^{*}<g^{L F}$. The laissez-faire growth rate will be larger than the optimal rate the stronger is the impact of the environmental externality on welfare (the smaller is $\kappa$ ).

The comparison of the share of the clean $\left(Y_{L} / Y\right)$ and dirty goods $\left(Y_{Z} / Y\right)$ in the final output in the laissez-faire equilibrium and the optimum is shown in the following Proposition.

Proposition 4 The value of $Y_{L} / Y$ in the long run of the laissez-faire equilibrium is lower than the optimal one, and the opposite occurs with $Y_{Z} / Y$. Also, the more consumers value the environment (the higher $\mu$ ), the larger is the difference between the laissez-faire and the optimal shares mentioned above. Also, emissions per unit of output are constant along the BGP and

\footnotetext{
${ }^{13}$ Jones and Williams [13] present empirical evidence, using econometric estimates of returns to $R \& D$, that socially optimal $R \& D$ investment is at least four times greater than actual spending (accounting for the current patent system and subsidies to research). Jones and Williams [14], using a calibrated model, and in the absence of taxes and subsidies, confirm the result of underinvestment in $\mathrm{R} \& \mathrm{D}$, not taking into account environmental quality.
} 
are higher in the laissez-faire economy than in the optimal solution according to

$$
\left(\frac{E_{Z}}{Y}\right)^{*}=\delta \frac{Y_{Z}}{Y}=\delta \kappa_{s}^{\gamma}\left(\frac{1-\gamma}{\gamma}\right)^{\gamma}\left(\frac{\eta_{Z} Z}{\eta_{L} L}\right)^{\beta^{\gamma}}=\kappa_{s}^{\gamma}\left(\frac{E_{Z}}{Y}\right)^{L F}
$$

Moreover, the more consumers value the environment (higher $\mu$ ), the lower is the optimal emissions intensity of final output.

Proof. See Appendix B.

Summarizing, for a given $\left(N_{Z} / N_{L}\right)$, the efficient relative production of the polluting good, and thus, the efficient level of emissions intensity of final output, is achieved by adjusting the production of intermediate inputs. The laissez-faire relative demand for dirty complementary intermediates is greater than the efficient one, as shown in (41) and (16). With endogenous technology, the internalization of the pollution externality also implies moving R\&D toward new clean complementary intermediates.

\section{$5 \quad$ Policy Implications}

We first define the first-best optimal policy, which works as a benchmark. Then, we look at second-best policy, assuming that the government is restricted to only one policy instrument when the optimal policy would require several.

\subsection{First-best policy}

The optimal policy is summarized in the following Proposition.

Proposition 5 The social optimum can be implemented through (i) a subsidy to the REBD sector given by $\phi^{*}=1 / \psi=1 /(1-\beta)$, (ii) a subsidy to the producers of intermediate inputs given by $s^{*}=\beta$, and, (iii) a tax on 
emissions given by

$$
\begin{aligned}
\tau^{*} & =\frac{\mu}{\delta(1-\gamma)} \frac{C}{Y} \\
& =\frac{\mu \rho}{\delta(1-\gamma)\left[(1-\mu)\left(\frac{1}{\psi}\right)^{\frac{1}{\beta}}\left(\gamma^{\gamma}(1-\gamma)^{1-\gamma}\right)^{\frac{1}{\beta}} \kappa_{s}^{\frac{1-\gamma}{\beta}}\left(\eta_{Z} Z\right)^{1-\gamma}\left(\eta_{L} L\right)^{\gamma}+\mu \rho\right]}
\end{aligned}
$$

Proof. Comparing the policy-ridden solution given by (19) with the social planner's solution (42), the results follow. $\kappa_{s}$ is the solution for equation (48) in the Appendix, and $\frac{\partial \kappa_{s}}{\partial \mu}<0$ holds.

The optimal tax increases with $\mu$ and decreases with the elasticity of $Y$ with respect to the polluting good, $\gamma$.

The first-best policy, summarized in the previous Proposition, implements not only the optimal growth rate of the economy, but also the optimal emissions intensity of final output, as it induces the efficient demand for intermediates and the efficient dirty-bias in the economy. The environmental tax decreases the demand for the dirty intermediates and, therefore, depresses the value of patents of new dirty complementary intermediates, encouraging environmentally oriented research. Since R\&D firms develop new clean or new dirty complementary intermediates depending on relative profitability, research toward new clean complementary intermediates is encouraged, thus, increasing the clean-bias in the economy toward its efficient level. $R \& D$ effort is optimally reallocated across sectors in response to the environmental tax. Therefore, the optimal level of emissions per unit of output is achieved.

\subsection{Second-best policy}

We now look at second-best policy. As the experience with climate negotiations shows, it has been difficult for nations to engage in a significant en- 
vironmental policy intervention. In this second-best setting, policy targeted to create incentives for technological change can be justified on economic grounds. Moreover, policies targeted to subsidizing technology have in general more political support than policies that increase costs, as the direct benefits are focused and the costs are dispersed, while they contribute to boosting growth. On the other hand, given the difficulty of governments in committing at present to sufficiently stringent enough environmental policy targets in anticipation of future emissions policy, to rely on technological policy makes it possible to undertake current commitments.

We look at each instrument separately below. Assuming that each governmental department establishes its own policy to pursue a given goal, perhaps responding to different lobbies, the interaction between them is not very likely to be taken into account when setting the level of each instrument. In this context, we examine the side effects of the different instruments when each one is decided independently of the others. Two kinds of instruments are considered: a tax on the polluting good and subsidies to the research sectors.

(1) Tax on the polluting good

Let us consider an emission tax, $\tau$, on the polluting sector to control emissions intensity. For $g^{L F}<g^{*}$, a positive tax on the polluting good further increases the gap between the two growth rates. However, for $g^{L F}>$ $g^{*}$ we may derive the tax rate for which the two growth rates are equated, as follows

$$
\tau=\left[\frac{1}{\delta}-\frac{1}{\delta}\left(\frac{1}{\psi}\right)^{\frac{1}{1-\gamma}} \kappa_{s}\right]>0
$$

Since this second-best tax is lower than the first-best one, the efficient level of emission intensity is not achieved. Thus, despite the fact that the 
economy is moving in the right direction, decreasing the relative production of $Y_{Z}$, and the level of emissions intensity when compared to the laissez-faire, pollution still remains above the efficient level.

Hence, for emissions pricing alone to provide sufficient incentive to implement the efficient dirty-bias, and eventually the efficient level of emissions intensity, the level of the tax has to be larger, implying more serious consequences to growth. Therefore, such policies have little political appeal.

(2) Subsidy to RED

If $g^{L F}>g^{*}$ any positive subsidy increases the gap between the two growth rates. In contrast, if $g^{L F}<g^{*}$, it is possible to achieve the optimal growth.

(i) Considering a technology policy that subsidizes both R\&D sectors at the same rate, that is, $\left(\phi=\phi_{Z}=\phi_{L}\right)$, growth is boosted, as mentioned above. In this case, the optimal growth rate of the economy can be implemented through

$$
\phi=\left[\left(\frac{1}{\psi}\right)^{\frac{1}{\beta}} \kappa^{\frac{1-\gamma}{\beta}}\right]>1 .
$$

However, since the introduction of $\phi$ does not require any adjustment on the demand of intermediate inputs, for a given $\left(N_{Z} / N_{L}\right)$, nor on the efficient dirty-bias, the relative production of $Y_{Z}$ is the same as in the laissez-faire economy. Thus, emissions per unit of output are the same as in the laissezfaire economy, above the efficient level. Notice that this is always the case, as long as the subsidies are the same between sectors, that is, independently of the rate.

(ii) In contrast to the previous case, the government's technology policy may discriminate between the new designs by paying a different subsidy to each sector, that is, $\phi_{Z} \neq \phi_{L}$. By computing the growth rate of the regulated 
economy, the following relationship between $\phi_{Z}$ and $\phi_{L}$ must hold

$$
\phi_{Z}=\left[\left(\frac{1}{\psi}\right)^{\frac{1}{\beta}} \kappa_{s}^{\frac{1-\gamma}{\beta}} \phi_{L}^{-\gamma}\right]^{\frac{1}{1-\gamma}}
$$

in order to implement the optimal growth rate. Also, if $\phi_{Z}=\kappa_{s} \phi_{L}$ holds, the efficient dirty-bias is implemented. Solving this system of equations, we obtain $\phi_{Z}=\left(\frac{1}{\psi}\right)^{\frac{1}{\beta}} \kappa_{s}^{\frac{1-\gamma}{\beta}+\gamma}$ and $\phi_{L}=\left(\frac{1}{\psi}\right)^{\frac{1}{\beta}} \frac{\frac{(1-\gamma)(1-\beta)}{\beta}}{\kappa_{s}}$, for which both the optimal growth rate of the economy and the efficient dirty-bias in the economy are obtained. Since $\kappa_{s}<1, \phi_{Z}<\phi_{L}$, the new $Z$-complementary intermediates become relatively more costly than the new $L$-complementary intermediates. This will encourage research toward new clean complementary intermediates, thereby increasing the clean-bias in the economy.

Discriminating between R\&D subsidies in favor of the clean sector corrects for the technology externalities and at the same time reduces emissions. However, there is no adjustment on the demand of intermediate inputs, for a given $\left(N_{Z} / N_{L}\right)$. Moreover, after endogenizing for technical change, the relative demand of dirty complementary intermediates is higher than the optimal one. Therefore, although the efficient dirty-bias is implemented, the relative production of $Y_{Z}$ is greater than the efficient one, and, consequently, so is the emissions intensity. However, the economy moves toward the optimum, that is, in the right direction, and produces a lower share of emissions in the final output than in the laissez-faire equilibrium.

\section{Conclusions}

In this paper we develop an endogenous growth model that incorporates the welfare effects of environmental quality and innovation. The final output is produced from two goods and two factors of production, one clean and one 
dirty. There are two different R\&D sectors associated with each sector of production. Technological change extends the range of two types of intermediate inputs: clean and dirty complementary intermediates. Production of the dirty intensive good yields emissions that decrease environmental quality and, therefore, consumers' utility.

We describe the regulated economy and compare the optimum to the laissez-faire regime. The laissez-faire growth rate can be higher or lower than the optimal growth rate since the environmental externality and the monopoly and R\&D distortions have opposite effects. We have shown that neither the rate of innovation nor the research effort directed at new clean complementary intermediates in the market economy are optimal. For this reason, the dirty-bias of technology in the laissez-faire economy is too high.

We compute the optimal values for the first-best policy tools: a subsidy to the $\mathrm{R} \& \mathrm{D}$ sector to encourage research, a subsidy to the producers of intermediate inputs to neutralize the effect of monopoly pricing, and an environmental tax on emissions to eliminate the pollution externality. We show that the optimal policy implements the efficient rate of innovation and also encourages environmentally oriented research in the regulated economy, reallocating $\mathrm{R} \& \mathrm{D}$ efforts across the two sectors.

In a second-best world we consider that each instrument is set independently. We consider a tax on emissions and a uniform and a differentiated subsidy to each $R \& D$ sector. Our results suggest that to achieve the efficient level of emissions intensity through pricing policies alone, the costs imposed are too high, as the price required to create incentives for clean technologies is too high, decreasing economic activity by more than desirable. Moreover, it has been shown in the literature that in order to provide incentives for 
firms to invest in the development of those technologies, credible commitments to meeting long-run emissions targets without imposing unnecessarily high near-term costs have to be provided. The fact that the caps to be set in the future are those that are relevant for innovation, and that it is not possible at present to credibly impose strict future targets gives additional importance to policies that increase government funding or incentives for private funding of research directed toward cleaner technologies (grants and tax incentives, prizes, R\&D consortia). In this case, we show that in an economy that cares about the quality of the environment it is better to discriminate between the two $\mathrm{R} \& \mathrm{D}$ sectors rather than to set a homogeneous subsidy to both sectors. However, we have shown that in order to achieve the efficient level of emissions intensity, besides subsidizing the two R\&D sectors differently, an additional tax on the polluting good is needed. 


\section{Appendix A - Endogeneity of $\kappa$}

Equation (24) can be rewritten as $\kappa=1-\frac{\mu}{(1-\gamma)} \frac{C}{Y}$. Thus, to obtain $\kappa$, we must derive the consumption to output ratio, $\frac{C}{Y}$, at the optimal solution.

Share of output devoted to consumption $(C / Y)^{14}$

In order to obtain $C / Y$, we need to make use of the final good resource constraint (5). From this condition, $\frac{C}{Y}=1-\frac{I}{Y}-\frac{R}{Y}$. Thus, we need to determine $I / Y$ and $R / Y$.

The total cost of producing $L$ and $Z$-complementary intermediates is $I=\psi x_{L}^{*}(j) N_{L}+\psi x_{Z}^{*}(j) N_{Z}$. Substituting (37) and (38) into the production functions (3), and solving for the total amount of intermediates purchased $\left(x_{i}^{*}(j) N_{i}\right)$, we obtain $x_{i}^{*}(j) N_{i}=\left(\frac{r_{i}}{p_{C} \psi}\right) Y_{i}(1-\beta)$. Together with (25) and (26), we obtain $I=(1-\beta) Y_{L} \frac{\partial Y}{\partial Y_{L}}+(1-\beta) Y_{Z}\left(\frac{\partial Y}{\partial Y_{Z}}-\mu \frac{C}{Y_{Z}}\right)$. As $Y=Y_{L} \frac{\partial Y}{\partial Y_{L}}+Y_{Z} \frac{\partial Y}{\partial Y_{Z}}$, the share of final good invested in intermediate inputs is given by $\frac{I}{Y}=(1-\beta)\left(1-\mu \frac{C}{Y}\right)$.

From (4), R\&D expenditures in each sector can be written as $R_{i}=$ $g^{*} N_{i} / \eta_{i}$. From (27), (29), (32), (34), (37) and the production function for $Y_{L}$ after substituting for $x_{L}^{*}$, we find that $\frac{N_{L}}{\eta_{L}}=\frac{Y_{L} \beta(1-\beta)}{g^{*}+\rho}\left(\frac{r_{L}}{p_{C} \psi}\right) \cdot \frac{N_{Z}}{\eta_{Z}}$ can be obtained similarly. Thus, total expenditure in $\mathrm{R} \& \mathrm{D}$ can be written as $R=$ $\frac{Y_{L} \beta g^{*}}{g^{*}+\rho} \frac{\partial Y}{\partial Y_{L}}+\frac{Y_{Z} \beta g^{*}}{g^{*}+\rho}\left(\frac{\partial Y}{\partial Y_{Z}}-\mu \frac{C}{Y_{Z}}\right)$ which implies that $\frac{R}{Y}=\frac{\beta g^{*}}{g^{*}+\rho}\left(1-\mu \frac{C}{Y}\right) .{ }^{15}$

Finally, from (5), and taking into account $g^{*}$, given by (42), we obtain

$$
\begin{aligned}
\frac{C}{Y} & =\frac{\beta-\frac{\beta g^{*}}{g^{*}+\rho}}{1-\mu\left[(1-\beta)+\frac{\beta g^{*}}{g^{*}+\rho}\right]} \\
& =\frac{\rho}{(1-\mu)\left(\frac{1}{\psi}\right)^{\frac{1}{\beta}}\left(\gamma^{\gamma}(1-\gamma)^{1-\gamma}\right)^{\frac{1}{\beta}} \kappa^{\frac{1-\gamma}{\beta}}\left(\eta_{Z} Z\right)^{1-\gamma}\left(\eta_{L} L\right)^{\gamma}+\mu \rho}
\end{aligned}
$$

\footnotetext{
${ }^{14}$ We follow Di Maria and Smulders [6], in a different context.

${ }^{15}$ It can be shown that $\frac{I}{Y}, \frac{R}{Y}$ and $\frac{C}{Y} \in(0,1)$.
} 
Substituting $\frac{C}{Y}$ in $\kappa$ (equation (24)), we get

$$
\kappa=1-\varphi(\kappa), \quad \text { where }
$$

$$
\varphi(\kappa)=\frac{\mu \rho}{(1-\gamma)(1-\mu)\left(\frac{1}{\psi}\right)^{\frac{1}{\beta}}\left(\gamma^{\gamma}(1-\gamma)^{1-\gamma}\right)^{\frac{1}{\beta}} \kappa^{\frac{1-\gamma}{\beta}}\left(\eta_{Z} Z\right)^{1-\gamma}\left(\eta_{L} L\right)^{\gamma}+(1-\gamma) \mu \rho}
$$

Let $\kappa=\left\{\kappa_{s}\right\}$ represent the set of solutions for this equation.

Lemma 1 At the equilibrium value of $\kappa_{s}, \frac{\partial \kappa_{s}}{\partial \mu}<0$

By analogy with stability analysis, we can show that $\frac{\partial\left(1-\varphi\left(\kappa_{s}\right)\right)}{\partial \kappa}<1$ iff $\frac{\partial \kappa\left(\kappa_{s}\right)}{\partial \mu}<0$. In other words, at the equilibrium value of $\kappa_{s}$, as $(1-\varphi(\kappa))$ is positively sloped, it has to cut the 45 -degree line from above.

$$
\begin{aligned}
& \text { Assume } A=\left(\frac{1}{\psi}\right)^{\frac{1}{\beta}}\left(\gamma^{\gamma}(1-\gamma)^{1-\gamma}\right)^{\frac{1}{\beta}}\left(\eta_{Z} Z\right)^{1-\gamma}\left(\eta_{L} L\right)^{\gamma} \\
& \frac{\partial\left(1-\varphi\left(\kappa_{s}\right)\right)}{\partial \kappa}<1 \Longleftrightarrow \frac{\partial \kappa\left(\kappa_{s}\right)}{\partial \mu}<0
\end{aligned}
$$

where

$$
\frac{\partial\left(1-\varphi\left(\kappa_{s}\right)\right)}{\partial \kappa}=\frac{(1-\gamma)^{2} A \beta^{-1} \frac{1-\mu}{\mu} \kappa_{s}^{\frac{1-\gamma}{\beta}-1} \rho}{\left[(1-\gamma) A \frac{1-\mu}{\mu} \kappa_{s}^{\frac{1-\gamma}{\beta}}+(1-\gamma) \rho\right]^{2}}
$$

and

$$
\frac{\partial \kappa\left(\kappa_{s}\right)}{\partial \mu}=\frac{-(1-\gamma) \mu^{-2} A \kappa_{s}^{\frac{1-\gamma}{\beta}} \rho}{\left[(1-\gamma) A \frac{1-\mu}{\mu} \kappa_{s}^{\frac{1-\gamma}{\beta}}+(1-\gamma) \rho\right]^{2}-(1-\gamma)^{2} A \beta^{-1} \frac{1-\mu}{\mu} \kappa_{s}^{\frac{1-\gamma}{\beta}-1} \rho}
$$

Notice that $\frac{\partial\left(1-\varphi\left(\kappa_{s}\right)\right)}{\partial \kappa}<1$ is equivalent to having the denominator of $\frac{\partial \kappa\left(\kappa_{s}\right)}{\partial \mu}$ positive. Since the numerator of $\frac{\partial \kappa\left(\kappa_{s}\right)}{\partial \mu}$ is negative, then $\frac{\partial \kappa\left(\kappa_{s}\right)}{\partial \mu}<0$. On the other hand, if $\frac{\partial \kappa\left(\kappa_{s}\right)}{\partial \mu}<0$, since the numerator is negative, then the denominator has to be positive, implying that $\frac{\partial\left(1-\varphi\left(\kappa_{s}\right)\right)}{\partial \kappa}<1$.

The solution can be shown graphically. When $1-\gamma \leqslant \beta$ (that is, when the share of $Y_{Z}$ on $Y$ is less than or equal to the share of $Z$ on $\left.Y_{Z}\right), 1-\varphi(\kappa)$ is concave. Figure 1 illustrates the left-hand side and the right-hand side of equation (48), for a given $\mu$, when $1-\gamma \leqslant \beta$. 
When $1-\gamma>\beta, 1-\varphi(\kappa)$ is convex for $\kappa<\hat{\kappa}$, and concave for $\kappa>\hat{\kappa} .^{16}$ Figure 2 illustrates this case.

In both cases, as $\mu$ increases, $1-\varphi(\kappa)$ crosses the horizontal axis at a higher $\kappa \cdot{ }^{17}$ Also, when $1-\gamma>\beta$, the inflection point, $\hat{\kappa}$, is increasing in $\mu$. Thus, the right-hand side of equation (48) moves to the right.

There is a solution for equation (48) as long as $\exists \kappa: 1-\varphi(\kappa)>\kappa$, that is, $(1-\gamma)(1-\mu)\left(\frac{1}{\psi}\right)^{\frac{1}{\beta}}\left(\gamma^{\gamma}(1-\gamma)^{1-\gamma}\right)^{\frac{1}{\beta}}\left(\eta_{Z} Z\right)^{1-\gamma}\left(\eta_{L} L\right)^{\gamma} \kappa^{\frac{1-\gamma}{\beta}}(1-\kappa)-(1-\gamma) \mu \rho \kappa-$ $\gamma \mu \rho>0$.

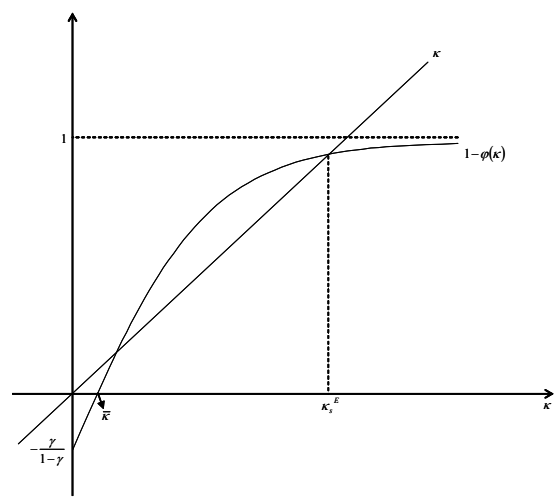

Figure 1: $\kappa=1-\varphi(\kappa)$ (eq. (48)), when $1-\gamma \leqslant \beta$

\section{Appendix B - Proof (Proposition 4)}

Notice that the share of the clean good in the final output is given by the inverse of $\frac{Y}{Y_{L}}=\left(\frac{Y_{Z}}{Y_{L}}\right)^{1-\gamma}$, that is, from (14), by $\frac{Y}{Y_{L}}=\left(\frac{1-\gamma}{\gamma}\left(\frac{\frac{\partial Y}{\partial Y_{Z}}}{\frac{\partial Y}{\partial Y_{L}}}\right)^{-1}\right)^{1-\gamma}$. After substituting for the efficient marginal rate of transformation between the two goods, with endogenous technical change (equation (40), and $\left(N_{Z} / N_{L}\right)$

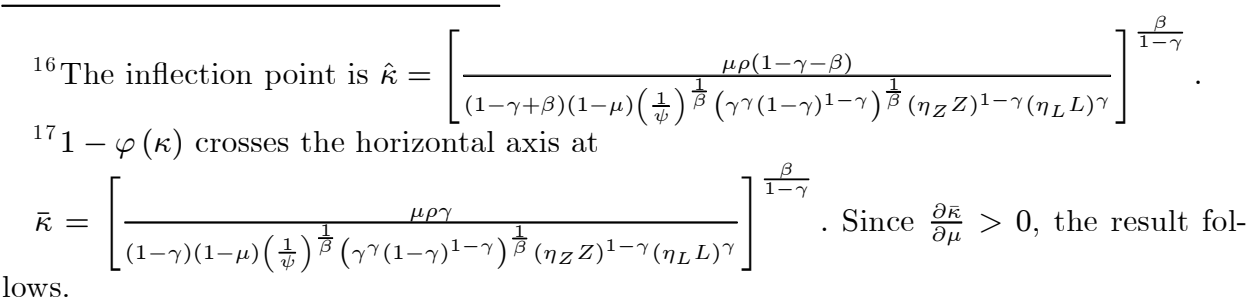




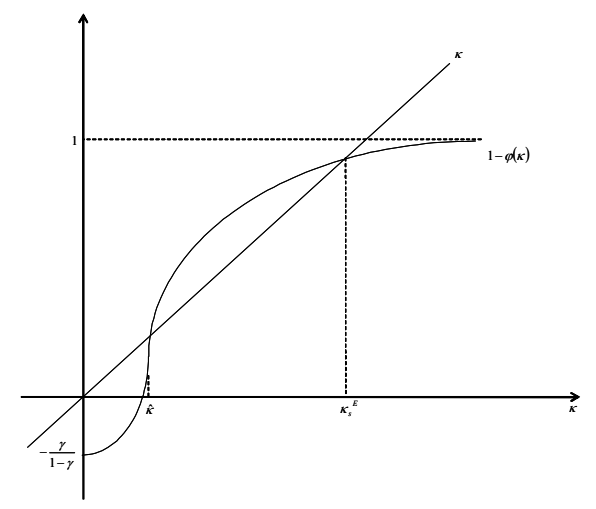

Figure 2: $\kappa=1-\varphi(\kappa)$ (eq. (48)), when $1-\gamma>\beta$

from equation (23)), we obtain $\left(\frac{Y}{Y_{L}}\right)^{*}=\left(\kappa_{s} \frac{1-\gamma}{\gamma}\left(\frac{\eta_{Z} Z}{\eta_{L} L}\right)^{\beta}\right)^{1-\gamma}=\kappa_{s}^{1-\gamma}\left(\frac{Y}{Y_{L}}\right)^{L F}$. Likewise, we have that $\left(\frac{Y}{Y_{Z}}\right)^{*}=\left(\frac{Y_{Z}}{Y_{L}}\right)^{-\gamma}=\left(\kappa_{s} \frac{1-\gamma}{\gamma}\left(\frac{\eta_{Z} Z}{\eta_{L} L}\right)^{\beta}\right)^{-\gamma}=\kappa_{s}^{-\gamma}\left(\frac{Y}{Y_{Z}}\right)^{L F}$. Since $0<\kappa_{s}<1$, it follows that $\left(\frac{Y}{Y_{L}}\right)^{*}<\left(\frac{Y}{Y_{L}}\right)^{L F}$ and $\left(\frac{Y}{Y_{Z}}\right)^{*}>\left(\frac{Y}{Y_{Z}}\right)^{L F}$. By differentiating $\frac{Y}{Y_{L}}$ and $\frac{Y}{Y_{Z}}$ with respect to $\mu$, it follows that $Y_{L}$ becomes relatively more important in the production of the final output the higher $\mu$ is, and the opposite occurs with $Y_{Z}$. 


\section{References}

[1] Acemoglu, D., 2002, "Directed Technical Change", Review of Economic Studies 69, 781-809.

[2] Acemoglu, D. and F. Zilibotti, 2001, "Productivity Differences", Quarterly Journal of Economics, 563-606.

[3] Amano, M. and R. A. Sedjo, 2006, "Forest Sequestration: Performance in Selected Countries in the Kyoto Period and the Potential Role of Sequestration in Post-Kyoto Agreements", Resources for the Future.

[4] Bovenberg, A. L. and S. Smulders, 1995, "Environmental Quality and Pollution-Augmenting Technological Change in a Two-sector Endogenous Growth Model", Journal of Public Economics 57, 369-391.

[5] Carraro, C. and D. Siniscalco, 1994, "Environmental Policy Reconsidered: The Role of Technological Innovation", European Economic Review 38, 545-555.

[6] Di Maria, C. and S. Smulders, 2004, "Trade Pessimists vs. Technology Optimists: Induced Technical Change and Pollution Havens", Advances in Economic Analysis 83 Policy, Vol. 4, No. 2, Article 7. http://www.bepress.com/bejeap/advances/vol4/iss2/art7

[7] Elbasha, E. H. and T. L. Roe, 1996, "On Endogenous Growth: The Implications of Environmental Externalities", Journal of Environmental Economics and Management 31, 240-268.

[8] Fisher, C. and R. Newell, 2008, "Environmental and Technology Policies for Climate Mitigation", Journal of Environmental Economics and Management 55, 142-162. 
[9] Grimaud, A., 1999, "Pollution Permits and Sustainable Growth in a Shumpeterian Model", Journal of Environmental Economics and Management 38, 249-266.

[10] Grimaud, A. and L. Rouge, 2008, "Environment, Directed Technical Change and Economic Policy", Environmental and Resource Economics 41, 439-463.

[11] Hart, R., 2004, "Growth, Environment and Innovation-A Model with Production Vintages and Environmentally Oriented Research", Journal of Environmental Economics and Management 48, 1078-1098.

[12] Hart, R., 2004, "Can Environmental Regulations Boost Growth?", Paper presented at SURED Conference, Ascona, Switzerland, June 2004.

[13] Jones, C. I. and J. C. Williams, 1998, "Measuring the Social Return to R\&D", The Quarterly Journal of Economics 113, 1119-1135.

[14] Jones, C. I. and J. C. Williams, 2000, "Too Much of a Good Thing? The Economics of Investment in R\&D", Journal of Economic Growth $\mathbf{5}, 65-85$.

[15] Montgomery, W. D. and A. E. Smith, 2007, "Price, Quantity and Technology Strategies for Climate Change Policy". In: Human-Induced Climate Change: An Interdisciplinary Assessment, Cambridge University Press, Cambridge.

[16] Reis, A., 2001, "Endogenous Growth and the Possibility of Eliminating Pollution", Journal of Environmental Economics and Management 42, 360-373. 
[17] Ricci, F., 2007, "Environmental Policy and Growth when Inputs are Differentiated in Pollution Intensity", Environmental and Resource Economics 38 (3), 285-310.

[18] Stokey, N., 1995, "R\&D and Economic Growth", Review of Economic Studies 62, 469-489.

[19] Stokey, N., 1998, "Are There Limits to Growth?", International Economic Review 39, 1-31. 


\section{Only for the referees,} Showing that $\frac{I}{Y}, \frac{R}{Y}$ and $\frac{C}{Y} \in(0,1)$.

The optimal share of output devoted to consumption, investment and $\mathrm{R} \& \mathrm{D} \in(0,1)$, as expected.

$$
\frac{C}{Y}=\frac{\beta-\frac{\beta g^{*}}{g^{*}+\rho}}{1-\mu\left[(1-\beta)+\frac{\beta g^{*}}{g^{*}+\rho}\right]}
$$

(i) The numerator is positive: $\beta-\frac{\beta g^{*}}{g^{*}+\rho}=\frac{\beta \rho}{g^{*}+\rho}>0$. Thus, $\frac{C}{Y}>0$, as long as the denominator $1-\mu\left[(1-\beta)+\frac{\beta g^{*}}{g^{*}+\rho}\right]=\frac{\left(g^{*}+\rho\right)(1-\mu)+\mu \beta \rho}{g^{*}+\rho}>0$, which is true if $\mu<1$.

(ii) $\frac{C}{Y}<1$ if $\beta-\frac{\beta g^{*}}{g^{*}+\rho}<1-\mu\left[(1-\beta)+\frac{\beta g^{*}}{g^{*}+\rho}\right]$, that is, $\frac{\beta \rho-\left(g^{*}+\rho\right)+\mu\left[(1-\beta)\left(g^{*}+\rho\right)+\beta g^{*}\right]}{g^{*}+\rho}<$ 0 . Since $g^{*}+\rho>0$, the numerator must be negative. After some algebra, it can be rewritten as $\beta \rho-\left(g^{*}+\rho\right)<0$. Since $\beta \in(0,1), \beta \rho<\rho$ holds. Thus, $\beta \rho<g^{*}+\rho$ also holds because $g^{*}>0$.

(iii) Also, $1-\mu \frac{C}{Y}>0$, because $\frac{\mu \beta-\frac{\mu \beta g^{*}}{g^{*}+\rho}}{1-\mu\left[(1-\beta)+\frac{\beta g^{*}}{g^{*}+\rho}\right]}<1 \Longleftrightarrow 1-\mu>0$.

$$
\frac{I}{Y}=(1-\beta)\left(1-\mu \frac{C}{Y}\right)
$$

(i) This ratio is positive because $\beta \in(0,1)$ and $1-\mu \frac{C}{Y}>0$.

(ii) Also, $\frac{I}{Y}<1$, because $\beta \in(0,1)$ and $0<\mu \frac{C}{Y}<1$.

$$
\frac{R}{Y}=\frac{\beta g^{*}}{g^{*}+\rho}\left(1-\mu \frac{C}{Y}\right)
$$

(i) This ratio is positive because $\frac{\beta g^{*}}{g^{*}+\rho}>0$ and $1-\mu \frac{C}{Y}>0$.

(ii) Also, $\frac{R}{Y}<1$, because $\beta g^{*}<g^{*}+\rho$ and $0<\mu \frac{C}{Y}<1$.

Proof (Comparative statics - Proposition 3) - Only for the referees

$$
\text { Assume } A=\left(\frac{1}{\psi}\right)^{\frac{1}{\beta}}\left(\gamma^{\gamma}(1-\gamma)^{1-\gamma}\right)^{\frac{1}{\beta}}\left(\eta_{Z} Z\right)^{1-\gamma}\left(\eta_{L} L\right)^{\gamma} \text {. }
$$


As shown in Appendix A: $\frac{\partial \kappa}{\partial \mu}=\frac{-(1-\gamma) \mu^{-2} A \kappa^{\frac{1-\gamma}{\beta}} \rho}{\left[(1-\gamma) A \frac{1-\mu}{\mu} \kappa^{\frac{1-\gamma}{\beta}}+(1-\gamma) \rho\right]^{2}-(1-\gamma)^{2} A \beta^{-1} \frac{1-\mu}{\mu} \kappa^{\frac{1-\gamma}{\beta}-1} \rho}=$ $=\frac{\rho A \kappa^{\frac{1-\gamma}{\beta}}}{(1-\mu) \mu \rho \frac{1-\gamma}{\beta} A \kappa^{\frac{1-\gamma}{\beta}-1}-(1-\gamma)\left[(1-\mu) A \kappa^{\frac{1-\gamma}{\beta}}+\mu \rho\right]^{2}}<0$

Differentiating $g^{*}$ with respect to the relevant variable yields:

(i) $\frac{\partial g^{*}}{\partial L}=\beta A \frac{1-\gamma}{\beta} \kappa^{\frac{1-\gamma}{\beta}-1} \frac{\partial \kappa}{\partial L}+\beta A \kappa^{\frac{1-\gamma}{\beta}} \gamma\left(\eta_{L} L\right)^{-1} \eta_{L}>0$ because $\frac{\partial \kappa}{\partial L}=\frac{-\mu \rho(1-\mu) A \kappa^{\frac{1-\gamma}{\beta}} \gamma\left(\eta_{L} L\right)^{-1} \eta_{L}}{(1-\mu) \mu \rho \frac{1-\gamma}{\beta} A \kappa^{\frac{1-\gamma}{\beta}-1}-(1-\gamma)\left[(1-\mu) A \kappa^{\frac{1-\gamma}{\beta}}+\mu \rho\right]^{2}}>0$

$\frac{\partial g^{*}}{\partial Z}=\beta A \frac{1-\gamma}{\beta} \kappa^{\frac{1-\gamma}{\beta}-1} \frac{\partial \kappa}{\partial Z}+\beta A \kappa^{\frac{1-\gamma}{\beta}}(1-\gamma)\left(\eta_{Z} Z\right)^{-1} \eta_{Z}>0$ because

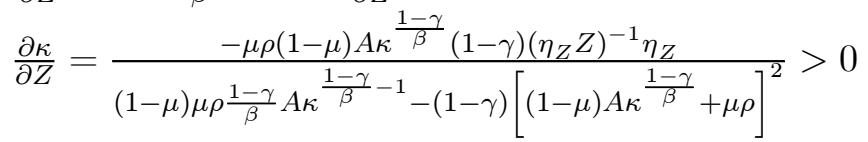

(ii) $\frac{\partial g^{*}}{\partial \eta_{L}}=\beta A \frac{1-\gamma}{\beta} \kappa^{\frac{1-\gamma}{\beta}-1} \frac{\partial \kappa}{\partial \eta_{L}}+\beta A \kappa^{\frac{1-\gamma}{\beta}} \gamma\left(\eta_{L} L\right)^{-1} L>0$ because

$\frac{\partial \kappa}{\partial \eta_{L}}=\frac{-\mu \rho(1-\mu) A \kappa^{\frac{1-\gamma}{\beta}} \gamma\left(\eta_{L} L\right)^{-1} L}{(1-\mu) \mu \rho \frac{1-\gamma}{\beta} A \kappa^{\frac{1-\gamma}{\beta}-1}-(1-\gamma)\left[(1-\mu) A \kappa^{\frac{1-\gamma}{\beta}}+\mu \rho\right]^{2}}>0$

$\frac{\partial g^{*}}{\partial \eta_{Z}}=\beta A \frac{1-\gamma}{\beta} \kappa^{\frac{1-\gamma}{\beta}-1} \frac{\partial \kappa}{\partial \eta_{Z}}+\beta A \kappa^{\frac{1-\gamma}{\beta}}(1-\gamma)\left(\eta_{Z} Z\right)^{-1} Z>0$ because

$\frac{\partial \kappa}{\partial \eta_{Z}}=\frac{-\mu \rho(1-\mu) A \kappa^{\frac{1-\gamma}{\beta}}(1-\gamma)\left(\eta_{Z} Z\right)^{-1} Z}{(1-\mu) \mu \rho \frac{1-\gamma}{\beta} A \kappa^{\frac{1-\gamma}{\beta}-1}-(1-\gamma)\left[(1-\mu) A \kappa^{\frac{1-\gamma}{\beta}}+\mu \rho\right]^{2}}>0$

(iii) $\frac{\partial g^{*}}{\partial \rho}=\beta A \frac{1-\gamma}{\beta} \kappa^{\frac{1-\gamma}{\beta}-1} \frac{\partial \kappa}{\partial \rho}-1<0$ because

$\frac{\partial \kappa}{\partial \rho}=\frac{\mu(1-\mu) A \kappa^{\frac{1-\gamma}{\beta}}}{(1-\mu) \mu \rho \frac{1-\gamma}{\beta} A \kappa^{\frac{1-\gamma}{\beta}-1}-(1-\gamma)\left[(1-\mu) A \kappa^{\frac{1-\gamma}{\beta}}+\mu \rho\right]^{2}}<0$

(iv) $\frac{\partial g^{*}}{\partial \mu}=\beta A \frac{1-\gamma}{\beta} \kappa^{\frac{1-\gamma}{\beta}-1} \frac{\partial \kappa}{\partial \mu}<0$ because $\frac{\partial \kappa}{\partial \mu}<0$ 